\title{
COMPLETING THE LOCAL SAMPLE WITH TYCHO
}

\author{
R. L. SMART, R. PANNUNZIO, M. G. LATTANZI \\ Osservatorio Astronomico di Torino, Italy \\ AND \\ B. MCLEAN \\ Space Telescope Science Institute, USA
}

The Tycho Catalogue contains proper motions and low precision parallaxes with a median precisions of 25 mas, while having high precision $B$ and V magnitudes (median precision of 0.07 and 0.06 respectivly). The number of high parallax stars is quite unexpected, with over 30 previously unreferenced, non-Hipparcos, stars with a parallax $>500$ mas and over 2000 with a parallax $>250$ mas. While many of these are probably mistakes, the completeness limit of Tycho and the precise magnitudes provide us with a way to intelligently build a complete picture of the nearby star field. Catalogues of nearby stars provide us with many fundamental parameters in the field of stellar astronomy, but the completness of known stars within say even 8pcs is still underestimated by about 30\%, i.e., 50-60 systems (T.J. Henry, CSSS 8, ASP Conf Series 64, 1994).

In a new program at Torino we are using the Tycho Catalogue to intelligently build a list of possible candidates for a $10 \mathrm{pc}$ volume. Figure 1 shows that Tycho will contain basically all ZAMS to spectral type M3 within $10 \mathrm{pcs}, \mathrm{M} 5$ to $4 \mathrm{pcs}$ and M6 to 2pcs. A comparison of the photometric and Tycho distances provides a list free of giants and systems where binarity has affected the color. This list is then added to the Torino Parallax program where we expect to provide more precise parallaxes.

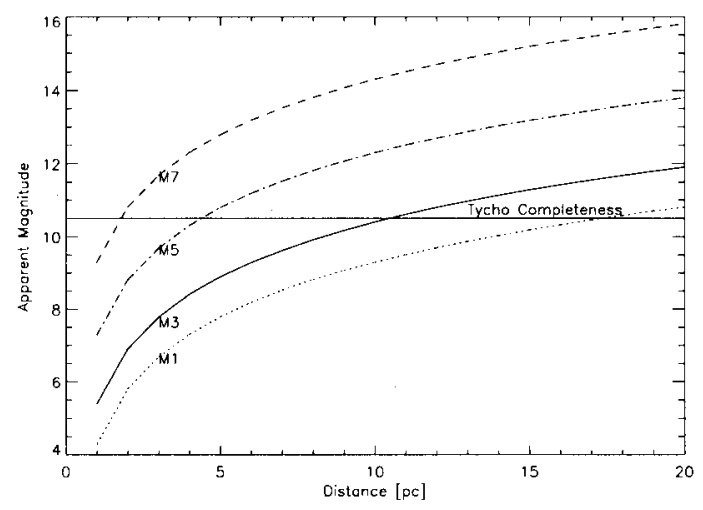

Figure 1. Change of observed magnitude with distance under the assumption of negligible absorption. The straight line indicates the approximate completeness limit of the Tycho catalogue. 